\title{
Adrenocorticotrophic hormone-stimulated cortisol release by the head kidney inter-renal tissue from sea bream (Sparus aurata) fed with linseed oil and soyabean oil
}

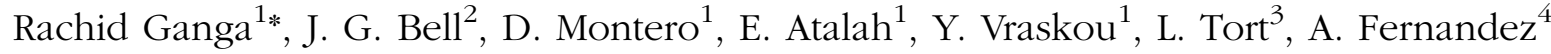 \\ and M. S. Izquierdo ${ }^{1}$ \\ ${ }^{1}$ Grupo de Investigación en Acuicultura, Universidad de Las Palmas de Gran Canaria E Instituto Canario de Ciencias \\ Marinas, Transmontaña s/n, 35416 Arucas, Las Palmas, Canary Islands, Spain \\ ${ }^{2}$ Institute of Aquaculture, University of Stirling, Stirling FK9 4LA, Scotland, UK \\ ${ }^{3}$ Departamento of Cell Biology, Physiology and Immunology, Universitat Autonóma de Barcelona, Bellaterra 08193, Spain \\ ${ }^{4}$ Biomar Iberia/ProAqua Nutrition, S.A., A-62, Km 99, ES-34210 Dueñas, Spain
}

(Received 10 February 2010 - Revised 23 June 2010 - Accepted 27 July 2010 - First published online 14 September 2010)

\section{Abstract}

The mode of action of highly unsaturated fatty acids (HUFA) in regulating gilthead sea bream (Sparus aurata) head kidney (HK) cortisol production was studied through in vitro trials using a dynamic superfusion system. Fish were previously fed with different diets containing several inclusion levels of linseed oil (LO) or soyabean oil (SO) for 26 weeks. Five diets were tested; anchovy oil was the only lipid source for the control diet (fish oil, FO) and two different substitution levels (70 and 100\%) were tested using either LO or SO (70LO, 70SO, 100LO and 100SO). Fatty acid compositions of the HK reflected the dietary input, thus EPA, DHA, arachidonic acid and $n$-3 HUFA were significantly $(P<0.05)$ reduced in fish fed vegetable oils compared with fish fed the FO diet. Feeding 70 or $100 \%$ LO increased significantly $(P<0.05)$ cortisol release in HK after stimulation with adrenocorticotrophic hormone (ACTH), while feeding SO had no effect on this response. Cortisol stimulation factor (SF) was increased in fish fed the 70LO and 100LO diets compared with fish fed the control diet. Moreover, eicosanoid inhibition by incubating the HK tissue with indomethacin (INDO) as a cyclo-oxygenase (COX) inhibitor, or nordihydroguaiaretic acid (NDGA) as a lipoxygenase (LOX) inhibitor, significantly reduced $(P<0 \cdot 05)$ the cortisol release after ACTH stimulation in the 70LO and 100LO diets. Cortisol SF was reduced in the FO, 70LO and 100LO diets when incubating the HK with INDO or NDGA, while it was increased in the $70 \mathrm{SO}$ diet. The present results indicate that changing the fatty acid profile of gilthead sea bream HK by including LO and/or SO in the fish diet affected the in vitro cortisol release, and this effect is partly mediated by COX and/or LOX metabolites.

Key words: Gilthead sea bream: Highly unsaturated fatty acids: Linseed oil: Soyabean oil: Indomethacin: Nordihydroguaiaretic acid: Cortisol: Head kidney: Superfusion

Marine teleosts have requirements for the essential longchain highly unsaturated fatty acids (HUFA) of the $n-3$ series, DHA $(22: 6 n-3)$ and EPA $(20: 5 n-3)^{(1-4)}$. Other studies have also pointed out the importance of the long-chain n-6 HUFA, arachidonic acid (ARA, 20:4n-6), as essential for marine fish ${ }^{(5,6)}$. These three fatty acids, as components of phospholipids, constitute a critical part of the cell membrane of most tissues and are responsible for maintaining an adequate physiological response of the cells ${ }^{(7,8)}$, being particularly important to promote stress resistance and defence against pathogenic challenge $^{(9)}$.

Stress in fish is monitored by levels of plasma cortisol, a general indicator of stressful conditions in vertebrates ${ }^{(10)}$, and its release into the circulation is controlled by the hypothalamus-pituitary-inter-renal axis. The cortisol release is preceded by the stimulation of the inter-renal tissue by secretion of pituitary hormones, in particular adrenocorticotrophic hormone (ACTH) ${ }^{(11)}$. Although some other hormones have been shown to stimulate cortisol

Abbreviations: ACTH, adrenocorticotrophic hormone; ARA, arachidonic acid; COX, cyclo-oxygenase; EFA, essential fatty acids; FO, fish oil; HK, head kidney; HUFA, highly unsaturated fatty acids; INDO, indomethacin; LA, linoleic acid; LNA, $\alpha$-linolenic acid; LO, linseed oil; LOX, lipoxygenase; NDGA, nordihydroguaiaretic acid; OA, oleic acid; SF, stimulation factor; SO, soyabean oil; VO, vegetable oils. 
release from inter-renal tissue, ACTH is the dominant secretagogue $^{(12-14)}$.

The understanding of the pathways and intracellular messengers that regulate cortisol synthesis in fish is very limited. The cortisol synthesis by ACTH has been found to be dependent on cyclic AMP as an intracellular second messenger in the coho salmon (Oncorbynchus kisutch) ${ }^{(15)}$. In mammals, the main pathway leading to corticosteroid synthesis by ACTH stimulation involves a signalling cascade integrating G-proteins, adenylyl cyclase, cyclic AMP and protein kinase $\mathrm{A}^{(16)}$. Other pathways, involving protein kinase $\mathrm{C}$ via stimulation by angiotensin II or acetylcholine, both known secretagogues of cortisol in fish ${ }^{(17)}$, share a role in the regulation of corticosteroid synthesis ${ }^{(18)}$. Moreover, protein kinase A has been suggested to be a crucial stimulatory component in the ACTH-mediated signalling pathway in fish adrenal steroidogenesis ${ }^{(19)}$, whereas protein kinase $\mathrm{C}$ has been shown to have an inhibitory role in the acute cortisol response in fish adrenocortical cells ${ }^{(19)}$.

The mechanism of how lipids and mainly HUFA could modulate cortisol release in vertebrates is not well known. In a previous study, we showed that free HUFA modulate cortisol secretion in sea bream head kidney (HK) maintained in superfusion ${ }^{(20)}$. ARA, EPA and DHA stimulated cortisol release by inter-renal tissue, while dihomo- $\gamma$-linolenic acid (20:3n-6) inhibited release. Besides, it was demonstrated that ACTH-induced cortisol release is partly mediated by cyclo-oxygenase (COX) metabolites ${ }^{(20)}$. Moreover, $\mathrm{PGE}_{2}$ derived from ARA has been shown to modulate the sensitivity of the hypothalamus-pituitary-adrenal axis, which is responsible for the release of cortisol in response to stress in mammals ${ }^{(21,22)}$ and possibly also in the homologous hypothalamuspituitary-inter-renal axis in fish ${ }^{(23)}$.

Fish oil (FO) has been the main source of HUFA in aquafeeds, but in recent years, due to the concerns about FO sustainability and cost, alternative oil sources are frequently included in fish feeds. Partial replacement of FO by vegetable oils (VO) does not affect fish growth or feed utilisation in several species ${ }^{(24-30)}$. Nevertheless, high substitution levels by VO have been shown to alter fish resistance to stressful conditions by increasing their cortisol levels ${ }^{(31)}$. However, little is known about the physiological mechanisms involved in the regulation of stress by fatty acids.

Since vegetable lipids lack HUFA, its dietary inclusion may modify tissue composition, altering cell membrane fluidity, receptor-mediated cortisol response and finally leading to a reduced functionality of the target organ. In addition, vegetable lipids can reduce the availability of eicosanoid precursors ARA, EPA, dihomo- $\gamma$-linolenic acid and DHA leading to dysfunctional eicosanoids signalling ${ }^{(32,33)}$ which may affect fish response to stress.

The aim of the present study was to clarify the effect of replacing FO by linseed oil (LO) and soyabean oil (SO), in the gilthead sea bream (Sparus aurata) stress response to ACTH stimulation. For this purpose, the HK tissue was maintained in a superfusion system and incubated with inhibitors of eicosanoid. Accordingly, five diets with different levels of FO substitution (0, 70 and 100), either with LO or with SO, were fed to gilthead sea bream juveniles.

\section{Materials and methods}

\section{Animals}

The study was carried out at Instituto Canario de Ciencias Marinas (Canary Islands, Spain), and fish were purchased from a local fish farm (ADSA, Canary Islands, Spain). A total of 750 gilthead sea bream ( $S$. aurata), with an average initial body weight of $45 \mathrm{~g}$, were randomly distributed in 500 litre polyethylene circular tanks (forty-five fish/tank, three tanks per diet). Tanks were supplied with continuous seawater $(36 \% 0)$ flow and aeration. Fish were reared under natural photoperiod conditions of approximately $12 \mathrm{~h}$ dark $-12 \mathrm{~h}$ light. Water temperature and dissolved $\mathrm{O}_{2}$ concentration during the experimental period ranged from $20 \cdot 0$ to $24 \cdot 2^{\circ} \mathrm{C}$ and from 5.04 to 8.7 parts per million, respectively.

\section{Diets}

Fish were fed the experiment diets until apparent satiation (three times/d, 6d/week), until they reached commercial market size after 26 weeks. Five isoenergetic and isonitrogenous experimental diets were formulated to provide a lipid content of $16 \%$. Anchovy oil was the only added lipid source in the FO diet (100\% FO). All the other diets contained VO to substitute either $70 \%$ of the anchovy oil by LO in 70LO diet, by SO in 70 SO diet or $100 \%$ of the anchovy oil by LO and SO in 100LO and $100 \mathrm{SO}$ diets, respectively. FO was included in 70LO and $705 \mathrm{~S}$ diets at a level high enough to meet the essential fatty acid (EFA) requirements of this species ${ }^{(34)}$.

\section{Biochemical analysis}

Lipid from the experimental diets and fish HK was extracted with chloroform-methanol $(2: 1, \mathrm{v} / \mathrm{v})$ as described previously $^{(35)}$. The fatty acid methyl esters were obtained by transesterification with $1 \% \mathrm{H}_{2} \mathrm{SO}_{4}$ in methanol and purified by absorption chromatography on $\mathrm{NH}_{2}$ Sep-pack cartridges (Waters Corporation, SA, Milford, MA, USA) and were separated and quantified by $\mathrm{GLC}^{(36)}$.

\section{Preparation and stimulation of head kidney tissue}

At the end of the feeding period, two fish were randomly taken from each tank in less than $1 \mathrm{~min}$, immediately anaesthetised with 2-phenoxyethanol (1:1000, v/v), and blood was collected with a hypodermic syringe from the 
caudal vein to minimise haemorrhage during the extraction of the tissue. HK tissue was removed from two fish in each superfusion trial, and in all series of experiments each treatment was assayed in quadruplicates (using two fish per replica, $n 8$ ), and cut into very small fragments in HEPES Ringer medium, which was used as the superfusion medium. Afterwards, HK homogenates were pooled and distributed in eight superfusion chambers (volume: $0.2 \mathrm{ml}$ ) in order to obtain a homogeneous aliquot in each of them. Tissues were superfused with a HEPES ( $\mathrm{pH} 7 \cdot 4)$ Ringer's solution containing $171 \mathrm{~mm}-\mathrm{NaCl}, 2 \mathrm{~mm}-\mathrm{KCl}$,

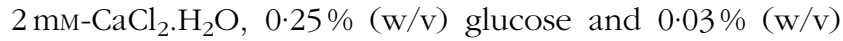
bovine serum albumin ${ }^{(37)}$. The system was temperature controlled at $18^{\circ} \mathrm{C}$, and the superfusion medium was pumped through the chamber at a rate of $75 \mathrm{ml} / \mathrm{min}$ by a Masterplex $\mathrm{L} / \mathrm{S}^{\mathrm{R}}$ multichannel peristaltic pump (Cole Parmer Intrument Company, Vernon Hills, IL, USA).

Trials were started after $3 \mathrm{~h}$ of superfusion when cortisol reached a stable baseline level ${ }^{(38,39)}$, in order to avoid deviations due to the different dispersion of interrenal cells in the perfusion preparation and the individual differences or the pre-stress level of each fish. After the stabilisation period of $3 \mathrm{~h}$, tissues were stimulated with ACTH at a concentration of $5 \mathrm{~nm}^{-\mathrm{hACTH}} \mathrm{TH}_{1-39}$ (Sigma, St Louis, MO, USA) for $20 \mathrm{~min}$. Subsequently, perfusion was maintained for another $170 \mathrm{~min}$, with fraction samples being collected every $20 \mathrm{~min}$ during this period. In a second series of experiments to clarify the action mechanisms of HUFA and the implication of eicosanoids in this process, the tissues were incubated with a COX inhibitor (indomethacin, INDO) or lipoxygenase (LOX) inhibitor (nordihydroguaiaretic acid, NDGA) for $20 \mathrm{~min}$ at a concentration of $25 \mu \mathrm{m}$ diluted in superfusion medium, and subsequently the tissues were stimulated with ACTH as explained before and maintained for another $170 \mathrm{~min}$, with samples being collected every $20 \mathrm{~min}$. Cortisol SF was calculated by comparing maximum cortisol released along the experiment after ACTH stimulation with baseline cortisol released (maximum release - baseline release) $\times 100$ / (baseline release) $^{(37)}$.

\section{Cortisol measurements}

Cortisol concentration in the perfused fluid was determined by RIA ${ }^{(37)}$. The antibody, Biolink, S.L. (Costa Mesa, CA, USA), was used in a final dilution of 1:6000. This antibody cross-reactivity is $100 \%$ with cortisol, $11 \cdot 40 \%$ with 21-deoxycorticosterone, 8.90\% with 11-deoxycortisol and $1.60 \%$ with 17 a-hydroxyprogesterone. The radioactivity was quantified using a liquid scintillation counter. Cortisol levels are expressed as ng/g HK per h.

Thiobarbituric acid-reactive substance analysis. Lipid peroxidation products were determined only in diets as thiobarbituric acid-reactive substances and were analysed according to the method described by Shahidi \& Hong ${ }^{(40)}$.

\section{Statistical analysis}

Significance of difference $(P<0.05)$ between dietary treatments was determined by one-way ANOVA followed by Duncan's multiple comparison test ${ }^{(41)}, n 8$. Analyses were performed using SPSS software (SPSS for windows 13; SPSS, Inc., Chicago, IL, USA).

\section{Results}

Effect of vegetable oil inclusion on fatty acid compositions

Thiobarbituric acid-reactive substances showed no significant difference between the diets, ranging between 8.56 and $3.85 \mathrm{mmol}$ of malondialdehyde/ kg of wet diet $(P<0.05)$, indicating no oxidation damage in the different diets.

Fatty acid composition of the diets reflected the inclusion of plant oils (Table 1). Total SFA ranged from

Table 1. Fatty acid profile of the experimental diets (\% total identified fatty acids)

\begin{tabular}{|c|c|c|c|c|c|}
\hline Fatty acids & FO & 70LO & 100LO & $70 S O$ & $100 S O$ \\
\hline $14: 0$ & 9.23 & $3 \cdot 11$ & 1.57 & 3.32 & 1.59 \\
\hline $15: 0$ & 0.26 & $0 \cdot 10$ & 0.06 & 0.11 & 0.06 \\
\hline $16:$ oISO & 0.11 & 0.04 & 0.03 & 0.05 & 0.03 \\
\hline $16: 0$ & $22 \cdot 21$ & $12 \cdot 19$ & $10 \cdot 21$ & $15 \cdot 84$ & 13.69 \\
\hline $16: 1 n-7$ & 11.25 & 3.91 & 1.98 & 4.06 & $2 \cdot 01$ \\
\hline $16: 1 n-5$ & 0.38 & 0.14 & 0.09 & 0.16 & 0.09 \\
\hline $16: 2 n-4$ & 1.83 & 0.61 & 0.28 & 0.61 & 0.29 \\
\hline $17: 0$ & 0.85 & 0.38 & 0.25 & 0.41 & 0.28 \\
\hline $16: 3 n-4$ & 2.00 & 0.66 & 0.29 & 0.67 & 0.31 \\
\hline $16: 3 n-3$ & 0.15 & 0.07 & 0.05 & 0.07 & 0.05 \\
\hline $16: 3 n-1$ & 0.12 & 0.05 & 0.04 & 0.06 & 0.04 \\
\hline $16: 4 n-3$ & 0.72 & 0.26 & 0.13 & 0.24 & 0.11 \\
\hline $18: 0$ & 3.85 & 3.96 & 3.99 & 3.43 & $3 \cdot 20$ \\
\hline $18: 1 n-9$ & $9 \cdot 10$ & 13.75 & $15 \cdot 31$ & $18 \cdot 60$ & 21.48 \\
\hline $18: 1 n-7$ & $3 \cdot 16$ & 1.57 & 1.36 & $2 \cdot 15$ & 1.77 \\
\hline $18: 1 n-5$ & 0.14 & 0.08 & - & 0.12 & 0.11 \\
\hline $18: 2 n-6$ & 4.06 & $12 \cdot 38$ & $16 \cdot 21$ & 29.93 & 38.51 \\
\hline $18: 2 n-4$ & 0.38 & 0.13 & 0.06 & 0.12 & 0.06 \\
\hline $18: 3 n-6$ & 0.36 & 0.13 & - & - & 0.07 \\
\hline $18: 3 n-4$ & 0.04 & - & 0.04 & 0.18 & 0.09 \\
\hline $18: 3 n-3$ & 0.48 & 31.94 & 37.63 & 5.63 & $6 \cdot 01$ \\
\hline $18: 4 n-3$ & 1.94 & 0.76 & 0.43 & 0.73 & 0.40 \\
\hline $20: 0$ & 0.28 & 0.21 & 0.19 & 0.28 & 0.28 \\
\hline $20: 1 n-9$ & 2.59 & 1.94 & 1.84 & 2.08 & 1.89 \\
\hline $20: 1 n-7$ & 0.26 & 0.13 & 0.09 & 0.13 & $0 \cdot 10$ \\
\hline $20: 2 n-9$ & - & 0.04 & - & 0.04 & 0.01 \\
\hline $20: 2 n-6$ & 0.19 & 0.11 & 0.08 & 0.11 & 0.09 \\
\hline $20: 3 n-6$ & 0.25 & 0.09 & 0.04 & 0.06 & 0.03 \\
\hline $20: 4 n-6$ & 1.11 & 0.43 & 0.24 & 0.43 & 0.24 \\
\hline $20: 4 n-3$ & 0.96 & 0.35 & 0.23 & 0.33 & $0 \cdot 16$ \\
\hline $20: 5 n-3$ & $10 \cdot 05$ & 4.00 & 2.07 & 3.77 & 2.06 \\
\hline $22: 1 n-11$ & 1.79 & 1.52 & 1.48 & 1.67 & 1.61 \\
\hline $22: 1 n-9$ & - & - & 0.42 & - & - \\
\hline $22: 4 n-6$ & 0.34 & 0.15 & 0.09 & 0.14 & 0.09 \\
\hline $22: 5 n-3$ & 1.74 & 0.66 & 0.29 & 0.60 & 0.29 \\
\hline $22: 6 n-3$ & 7.82 & $4 \cdot 16$ & 2.92 & 3.87 & $2 \cdot 91$ \\
\hline Saturates & 36.68 & 19.95 & $16 \cdot 28$ & 23.40 & $19 \cdot 10$ \\
\hline Monoenic & 28.79 & $23 \cdot 10$ & $22 \cdot 62$ & 29.03 & 29.09 \\
\hline$n-3$ & 23.42 & $42 \cdot 19$ & 43.72 & $15 \cdot 23$ & 12.00 \\
\hline$n-6$ & $6 \cdot 27$ & 13.26 & $16 \cdot 66$ & 30.67 & 39.02 \\
\hline$n-9$ & 22.99 & 19.66 & 19.55 & 24.78 & 25.40 \\
\hline$n-3$ HUFA & 20.57 & $9 \cdot 17$ & 5.50 & 8.57 & 5.42 \\
\hline$n-3: n-6$ & 3.74 & $3 \cdot 18$ & $2 \cdot 62$ & 0.50 & 0.31 \\
\hline
\end{tabular}

FO, fish oil; LO, linseed oil; SO, soyabean oil; HUFA, highly unsaturated fatty acids. 
$16 \cdot 28 \%$ in the $100 \mathrm{LO}$ diet to $36.68 \%$ in the FO diet. Total monoenoics fatty acids (mainly oleic acid (18: $1 n-9$, OA)) ranged from $22.62 \%$ in the $100 \mathrm{LO}$ diet to $29.09 \%$ in the $100 S O$ diet. $\alpha$-Linolenic acid (18:3n-3, LNA) increased with increasing inclusion of LO, from $0.48 \%$ in the FO diet to $37 \cdot 63 \%$ in the $100 \mathrm{LO}$ diet. Similarly, linoleic acid (18:2n-6, LA) increased, particularly with the increasing inclusion of SO, from $4.02 \%$ in the FO diet to $38.51 \%$ in the $100 \mathrm{SO}$ diet. EPA and DHA were reduced by the increased content of plant oils in the diets ranging from $10.05 \%$ in the FO diet to $2.06 \%$ in the $100 \mathrm{SO}$ diet and from $7 \cdot 82 \%$ in the FO diet to $2.91 \%$ in the $100 \mathrm{SO}$ diet, respectively. ARA was also decreased by the plant oil inclusion, ranging from $1 \cdot 11 \%$ in the FO diet to $0 \cdot 24 \%$ in the 100LO diet.

Fish HK fatty acid profile of total lipids reflected the dietary lipid composition (Fig. 1). n-3 fatty acids were significantly increased in fish fed the LO diet, while $n-6$ content was significantly increased in fish fed the SO diet. $n-9$ was also increased in fish fed the VO diet compared with fish fed the control diet, due mainly to OA increase. However, the content in HUFA (EPA, ARA and DHA), mainly $n-3$ series, was significantly $(P<0.05)$ reduced in fish fed the two VO, compared with fish fed the control diet, which was significantly reduced. Then a $45 \%$ reduction was found in DHA from the HK of fish fed the 70LO diet, $55 \%$ in fish fed the 100LO diet, $58 \%$ in fish fed the 70 SO diet and $61 \%$ in fish fed the $100 \mathrm{SO}$ diet compared with FO-fed fish. A 64\% reduction was found in EPA from the HK of fish fed the 70LO diet, $76 \%$ in fish fed the 100LO diet, $73 \%$ in fish fed the 70 SO diet and $80 \%$ in fish fed the $100 \mathrm{SO}$ diet compared with FO-fed fish. Regarding ARA, a 16\% reduction was found in fish fed the $70 \mathrm{LO}$ diet, and $47 \%$ reduction was found in fish fed the 100LO diet, $88 \%$ in fish fed the 70 SO diet and $86 \%$ in fish fed the $1005 \mathrm{O}$ diet. As a consequence, the ARA:EPA ratio was also markedly affected by the

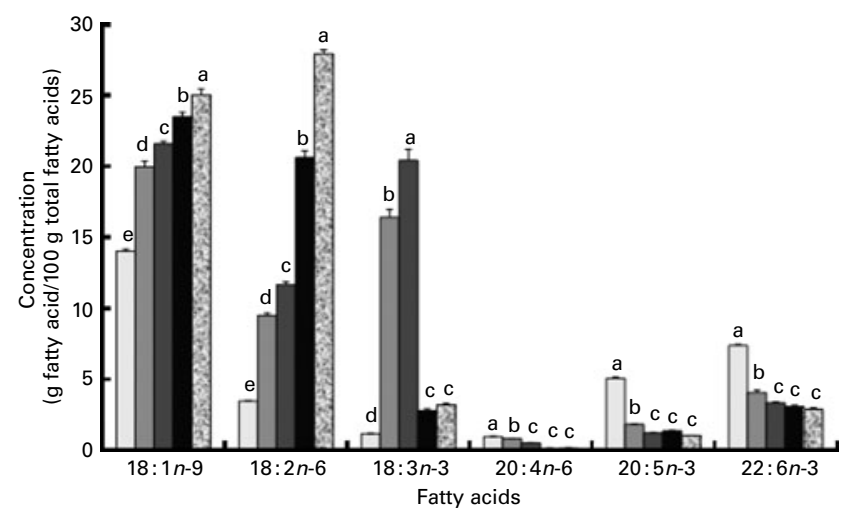

Fig. 1. Concentration (\% total fatty acids) of $18: 2 n-6,18: 3 n-3,18: 1 n-9$, $20: 4 n-6,20: 5 n-3$ and $22: 6 n-3$ in the head kidney of sea bream (Sparus aurata) fed either a fish oil (FO), linseed oil (LO) or soyabean oil (SO) diet for 26 weeks. Values are means. ${ }^{a, b, c, d, e}$ Mean values with unlike letters were significantly different $(P<0.05), n$ 8. ․ FO; ㄷ, 70LO; , 100LO; 口, 70SO; ⿷匚, 100 SO. inclusion of VO and differed depending on the dietary oil fed. Thus, the lowest ARA:EPA ratio was found in the HK of fish fed the $70 \mathrm{SO}$ diet, followed by the 100SO, FO, 70LO diets and, finally, the 100LO diet.

\section{Effect of feeding vegetable oils on cortisol response to adrenocorticotrophic hormone stimulation}

Feeding fish with LO increased cortisol release in HK tissue after ACTH stimulation; fish fed the 100LO diet showed the highest cortisol release at 310, 330, 370 and $390 \mathrm{~min}$ after ACTH stimulation, the cortisol response was characterised by a first peak at $330 \mathrm{~min}$ followed by a reduction at 350 and $370 \mathrm{~min}$ and a strongest rise at $390 \mathrm{~min}$. Fish fed the 70LO diet showed the same pattern with less intensity up to $370 \mathrm{~min}$, and the cortisol continued to decrease at $390 \mathrm{~min}$, indicating that the effect of LO was proportional to its content in the diet. However, fish fed the $70 \mathrm{SO}$ and $100 S O$ diets showed slightly lower, but NS, cortisol response after ACTH challenge (Fig. 2). Therefore, fish fed SO diets showed a tendency to inhibit cortisol response, whereas fish fed LO diets showed a significant enhancement of cortisol release.

The overall cortisol release after ACTH challenge expressed as cortisol SF (Fig. 3) was significantly highest in the 100LO diet followed by the 70LO diet. No significant differences were found in cortisol SF of fish fed SO and FO diets. The correlation factor between SF and ARA:EPA in HK was $98 \%$, reflecting the importance of both EPA and ARA as eicosanoid precursors on cortisol secretion.

\section{Implication of cyclo-oxygenase and lipoxygenase metabolites in cortisol release}

Cortisol release in the HK after ACTH challenge was affected by the incubation with eicosanoid inhibitors. Control fish decreased cortisol release after ACTH stimulation when the HK tissue was incubated with both the inhibitors (INDO or NDGA) (Fig. 4), denoting the implication of metabolites from both COX and LOX enzymes

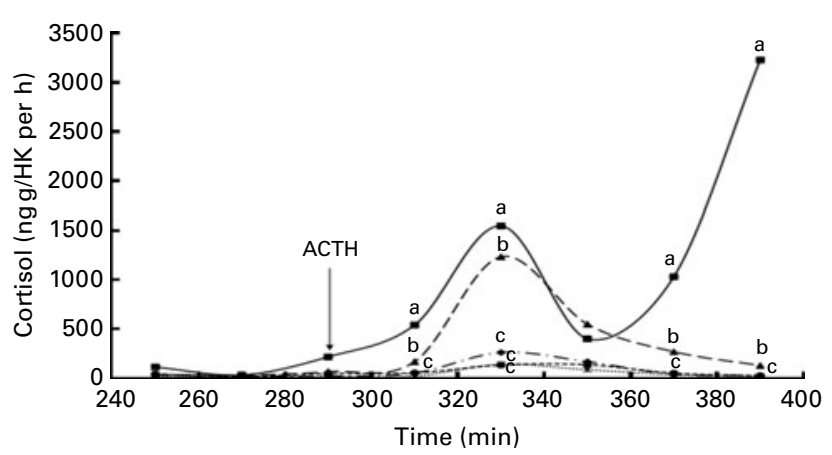

Fig. 2. Absolute cortisol secretion (ng/g head kidney per h) by head kidney of sea bream fed either fish oil (FO), linseed oil (LO) or soyabean oil (SO) after adrenocorticotrophic hormone (ACTH) stimulation. ${ }^{a, b, c}$ Mean values at the same time point with unlike letters were significantly different $(P<0.05)$,

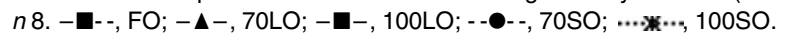




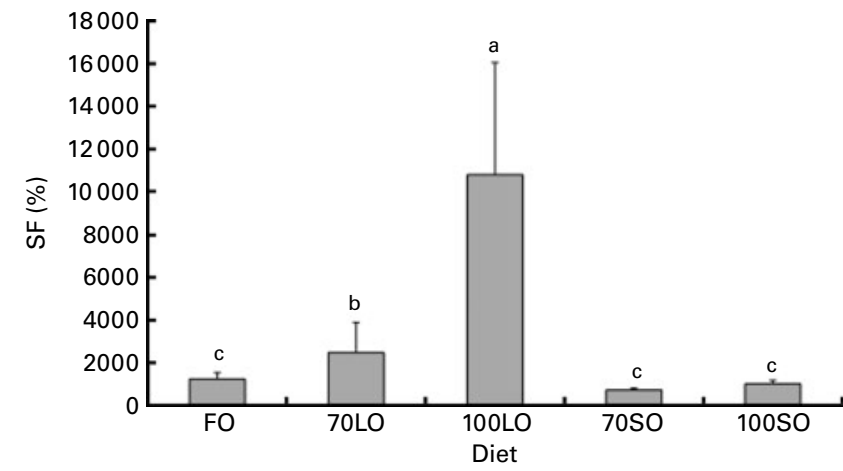

Fig. 3. Cortisol stimulation factor (SF) in head kidney of sea bream fed either fish oil (FO), linseed oil (LO) or soyabean oil (SO) after adrenocorticotrophic hormone stimulation. ${ }^{\mathrm{a}, \mathrm{b}, \mathrm{c}}$ Mean values with unlike letters were significantly different $(P<0.05), n 8$.

in cortisol secretion. When the HK tissue was incubated with INDO and NDGA, the cortisol release was significantly decreased in fish fed the $70 \mathrm{LO}$ diet, indicating the modulation of the cortisol secretion by these two pathways, with this decrease being more pronounced when NDGA was used as the inhibitor compared with INDO (Fig. 5).

In fish fed the 100LO diet, incubating the tissue with INDO or NDGA delayed the cortisol release, and the cortisol response was characterised by only one highest peak at 330 min. Incubating the tissue with NDGA decreased the cortisol release at $390 \mathrm{~min}$, proving again the role of COX and LOX in the stress secretion. Also in this case, the response was delayed, and the use of NDGA was more effective in inhibiting the cortisol release than that of INDO (Fig. 6). In fish fed the 70SO diet incubating the HK tissue with INDO increased cortisol release, indicating another pathway of action in this fish species. NDGA incubation did not affect cortisol release after ACTH stimulation (Fig. 7). HK tissue from fish fed the 100 SO diet showed also significantly decreased cortisol release after ACTH challenge when incubated with INDO, and there was

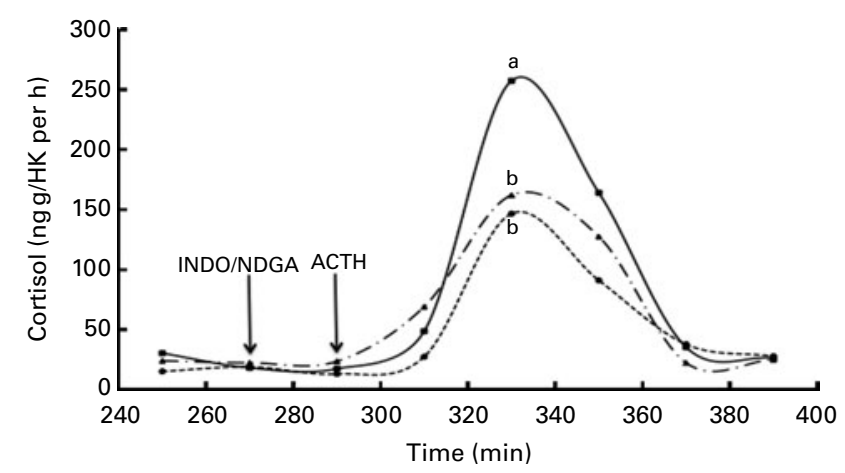

Fig. 4. Absolute cortisol secretion ( $\mathrm{ng} / \mathrm{g}$ head kidney per h) by head kidney of sea bream fed fish oil (FO) after adrenocorticotrophic hormone (ACTH) stimulation following incubation with either indomethacin (INDO) or nordihydroguaiaretic acid (NDGA). ${ }^{\mathrm{a}, \mathrm{b}}$ Mean values at the same time point with unlike letters were significantly different $(P<0.05), n 8 .-\mathbf{\square}-$, FO; --๑--, FO + INDO; - $\Delta--$, FO + NDGA.

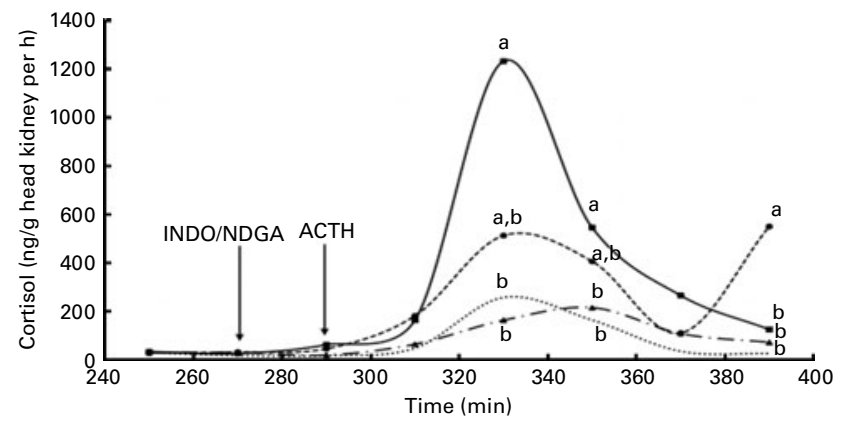

Fig. 5. Absolute cortisol secretion ( $\mathrm{ng} / \mathrm{g}$ head kidney per $\mathrm{h}$ ) by head kidney of sea bream fed 70 linseed oil (70LO) diet in comparison to the control diet (fish oil, FO) after adrenocorticotrophic hormone (ACTH) stimulation following incubation with either indomethacin (INDO) or nordihydroguaiaretic acid (NDGA). ${ }^{\text {a,b }}$ Mean values at the same time point with unlike letters were significantly different $(P<0.05), n$ 8. -,$-- 70 \mathrm{LO} ;--\bullet--, 70 \mathrm{LO}+$ INDO; - $\Delta--$, 70LO + NDGA; ..., FO.

also a slightly decreased cortisol release, but NS when the tissue was incubated with NDGA (Fig. 8).

Cortisol SF from the experiment using INDO and NDGA (Fig. 9) showed that incubating HK tissue with INDO in FO-fed fish reduced the cortisol SF to $26 \%$, and when incubated with NDGA, the SF was reduced to a $42 \%$. Also when the HK tissue in 70LO-fed fish was incubated with INDO or NDGA, the cortisol SF level was decreased by 39 and $66 \%$, respectively. HK tissue from 100LO-fed fish showed apparently the same level of decrease of the cortisol SF when incubated with INDO or NDGA corresponding to $46 \%$. By contrast, in fish fed the SO diet, the action of the COX and LOX inhibitors was different, such that incubating HK tissue from fish fed the $100 \mathrm{SO}$ diet, with INDO, reduced the cortisol SF to a $20 \%$, and the incubation with NDGA decreased the cortisol SF to only $10 \%$. By comparison, the fish fed the $70 \mathrm{SO}$ diet showed the opposite response to the use of COX and LOX inhibitors, as when the tissue was incubated with INDO, the cortisol SF increased by about 3-fold and when it was incubated with NDGA, the cortisol SF was increased by $60 \%$.

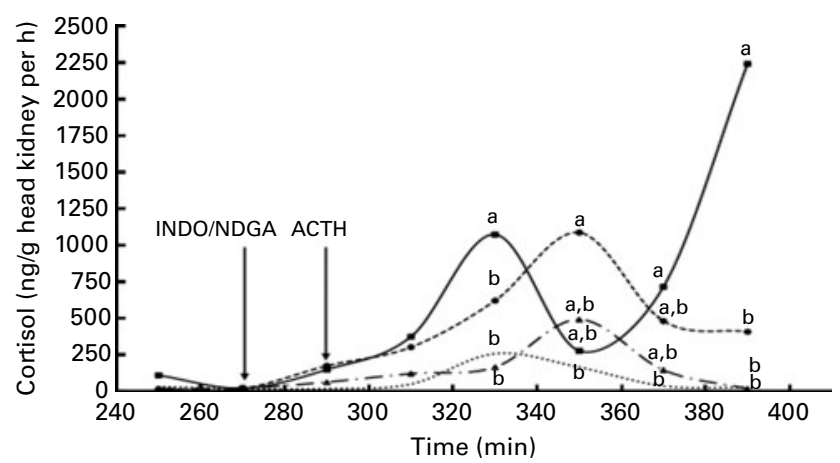

Fig. 6. Absolute cortisol secretion (ng/g head kidney per $\mathrm{h}$ ) by head kidney of sea bream fed 100 linseed oil (100LO) diet in comparison to the control diet (fish oil, FO) after adrenocorticotrophic hormone (ACTH) stimulation following incubation with either indomethacin (INDO) or nordihydroguaiaretic acid (NDGA). ${ }^{a, b}$ Mean values at the same time point with unlike letters were significantly different $(P<0.05), n$ 8. $-\mathbf{\square}-$, 100LO; --๑- -, 100LO + INDO; - $\Delta--$, 100LO + NDGA; ..., FO. 


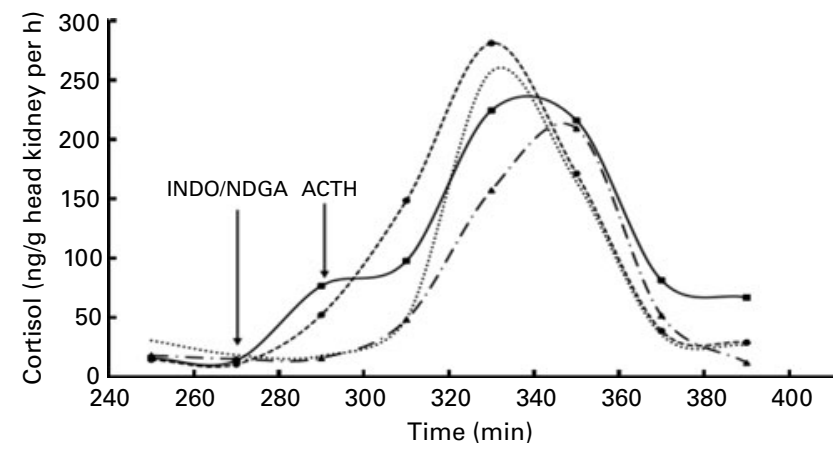

Fig. 7. Absolute cortisol secretion (ng/g head kidney per h) by head kidney of sea bream fed 70 soyabean oil (70SO) diet in comparison to the control diet (fish oil, FO) after adrenocorticotrophic hormone (ACTH) stimulation following incubation with either indomethacin (INDO) or nordihydroguaiaretic acid

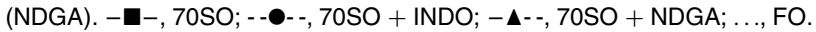

\section{Discussion}

The EFA for marine fish comprise HUFA with carbon chain lengths of 20 and 22, for both the $n-3$ and $n-6$ series. Because the HUFA cannot be synthesised 'de novo' by the fish ${ }^{(42)}$ at a sufficient rate to meet the requirements, these EFA must be provided in the diet. HUFA are essential for normal fish growth, for its cellular structure and functions, including the maintenance of membranes and eicosanoid metabolism ${ }^{(43)}$. Replacing FO, in commercial diets, with VO that are devoid of $n-3$ HUFA, resulted in reduced tissue levels of ARA, EPA and $\mathrm{DHA}^{(25,26,30,31,44)}$. In this experiment, at the end of the 26-week feeding period, the fatty acid profile of HK of total lipids from gilthead sea bream reflected the dietary input. Thus, fish fed the SO diet had significantly higher levels of LA and OA, and fish fed the LO diet had significantly higher levels of LNA and OA. In addition, since LO and SO contain large quantities of C18 fatty acids, the $n-6: n-3$ fatty acid ratio was altered, and this could affect fish health ${ }^{(30,31,45)}$. The contents of DHA and EPA decreased significantly in fish fed VO according to the level of inclusion, for instance,

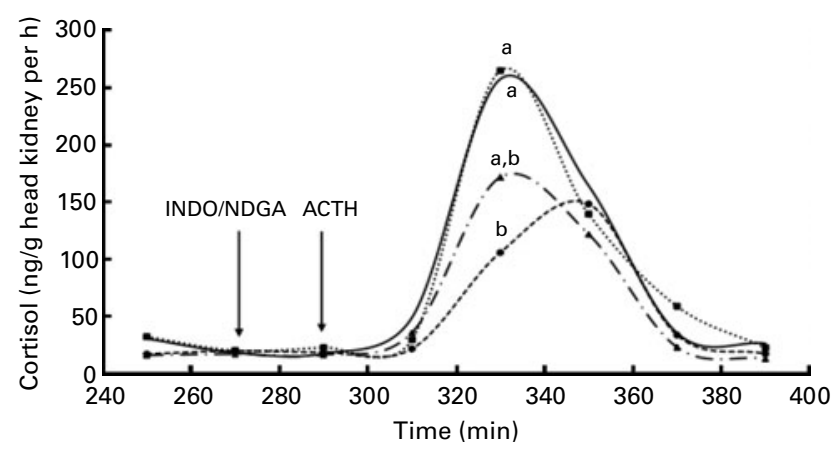

Fig. 8. Absolute cortisol secretion (ng/g head kidney per h) by head kidney of sea bream fed 100 soyabean oil (100SO) diet in comparison to the control diet (fish oil, FO) after adrenocorticotrophic hormone (ACTH) stimulation following incubation with either indomethacin (INDO) or nordihydroguaiaretic acid (NDGA). ${ }^{a, b}$ Mean values at the same time point with unlike letters were significantly different $(P<0.05), n$ 8. - - - , 100SO; -----, 100SO + INDO; $-\boldsymbol{\Delta}-\mathbf{-}, 100 \mathrm{SO}+\mathrm{NDGA} ;-$, FO.

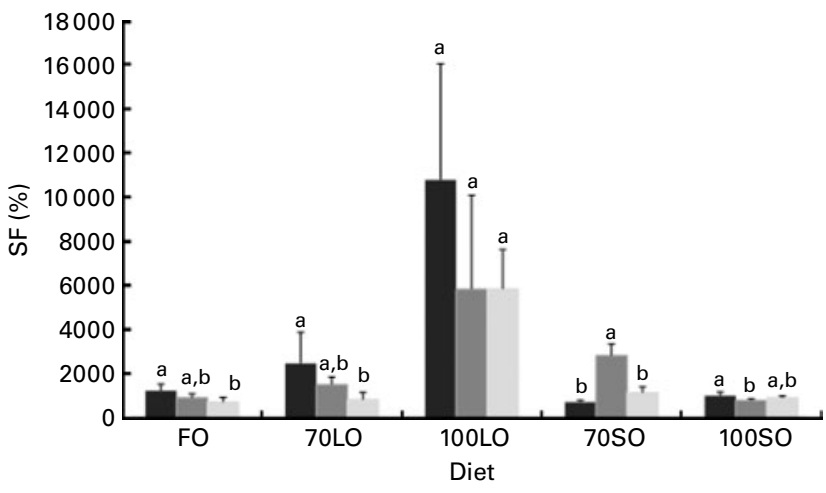

Fig. 9. Cortisol stimulation factor (SF) in head kidney of sea bream fed different diets after adrenocorticotrophic hormone (ACTH) stimulation following incubation with either indomethacin (INDO) or nordihydroguaiaretic (NDGA) acid. ${ }^{a, b}$ Mean values with unlike letters for each dietary treatment indicate significant difference due to INDO or NDGA addition $(P<0.05), n 8$. घ, Control; $\mathbf{a}$, INDO; , , NDGA.

EPA decreased by about $80 \%$ in fish fed the $100 \mathrm{SO}$ diet and $76 \%$ in fish fed the 100LO diet compared with fish fed the control diet, and DHA was decreased by $61 \%$ in fish fed the 100SO diet and 55\% in fish fed the 100LO. SFA and MUFA, particularly 16:0, 18:1n-9, $20: 1 n-9$ and $22: 1 n-11$, are easily catabolised in fish to produce energy, while DHA and EPA are less easily catabolised via $\beta$-oxidation ${ }^{(46)}$. Thus, an increase in the OA, LA and LNA contents in HK from fish fed VO may make these PUFA more available for oxidation to produce energy necessary to deal with the stress challenges.

Under stress conditions, cortisol is secreted from interrenal cells of the HK triggered by the corticotrophinreleasing hormone and ACTH hormonal cascade of the hypothalamus-pituitary-inter-renal axis in fish. ACTH is well known to induce the production and release of cortisol and is considered to be the major glucocorticoid stressor in fish ${ }^{(47)}$, playing an important regulatory role in the stress response process, especially in the metabolic adjustments to stress ${ }^{(48)}$. Feeding gilthead sea bream with the 70LO and 100LO diets significantly increased cortisol release from HK tissue after ACTH stimulation, in accordance with previous results from in vivo studies ${ }^{(31)}$. The levels of cortisol found in the present study for the control diet-fed fish were similar to those reported in our previous study ${ }^{(20)}$, whereas the results of HK from fish fed the 100LO diet were 9-fold higher than the control response, indicating an effect of including high levels of LO in diets for gilthead sea bream on its response to stress.

It is well established that HUFA provided by the diet play an important role in stress response in mammals as in fish. Enhancing the levels of dietary ARA promoted survival and resistance to stress in gilthead sea bream larvae ${ }^{(49,50)}$, and feeding the ARA-supplemented diet resulted in a diminution in cortisol response after net confinement compared with the fish fed a diet containing a low level of this fatty $\operatorname{acid}^{(51)}$. In the present study, the content of ARA in HK 
decreased by $55 \%$ in 70LO-fed fish, $81 \%$ in 100LO-fed fish, $86 \%$ in 70 SO-fed fish and $88 \%$ in 100 SO-fed fish, which could be associated partly with the modulation of cortisol release levels observed in these treatments. However, a number of authors have also demonstrated the importance of EPA and DHA in stress response and stimulating cortisol release in fish ${ }^{(9,20,52-54)}$. In this experiment, the low dietary levels of these important HUFA in the diet led to a parallel decrease in the HK causing a significant modulation of cortisol release. These results demonstrated that feeding fish with diets poor in these EFA affected cortisol release from HK after challenging the tissue with ACTH, clearly denoting the importance of these nutrients in the correct function of $\mathrm{HK}$ in response to stress situations.

In the literature, there is a lack of information on the effect of PUFA (of $n-3$ and or $n-6$ series) on cortisol release and stress response in fish. The apparent contradiction in cortisol release levels found between fish fed the LO and SO could be explained by the physiological role of different fatty acids provided by these oils. Once released from the membrane, NEFA could act directly as ligands, affecting positively or negatively the binding of steroid hormones to their specific intracellular receptors ${ }^{(55)}$. In addition, fatty acids can also co-regulate glucocorticoid-dependent gene expression by modulating the activity of protein kinases involved in the phosphorylating transcription factors ${ }^{(55)}$ Therefore, they may be playing a role in modulating the intracellular steroid hormone-signalling pathway to co-regulate a glucocorticoid-sensitive promoter ${ }^{(56)}$. In human platelets, unsaturated fatty acids such as palmitoleic acid (16:1), OA, LA, LNA and ARA were detected to inhibit phospholipase A2 activity ${ }^{(57)}$, which is the key enzyme responsible for liberating the COX and LOX substrate precursors from the membrane phospholipids.

The decrease in cortisol release in fish fed SO could be explained by the higher content of OA and LA in their tissue. In rats, the maximal steroidogenic response to ACTH was inhibited approximately to $50 \%$ by OA, concluding that the modulation of steroidogenesis by these abundant naturally occurring lipids may be an important component of the control mechanisms within the hypothalamus-pituitary-adrenal axis ${ }^{(58)}$. It was also demonstrated that OA and LA inhibited the action of ACTH on the adrenal gland ${ }^{(59)}$. Moreover, in human subjects, some reports indicated that increasing dietary $n-6: n-3$ fatty acids ratio by increasing the ratio between LA and LNA up to 4:1 reduced blood cortisol and cholesterol levels ${ }^{(60)}$. Elsewhere, LA was also reported to inhibit cortisol release in bovine adrenal cells ${ }^{(61)}$. By contrast, there was increased cortisol release in $\mathrm{HK}$ from fish fed LO rich in LNA after ACTH stimulation. Particularly, fish fed the 100LO diet registered significantly the highest cortisol level with low-peak response, the first one at $330 \mathrm{~min}$ and the maximum one at $390 \mathrm{~min}$; this may suggest the participation of other unknown mechanisms in such a response. These results are in accordance with a previous in vivo study, which showed that feeding sea bream with diets rich in LO increased significantly their plasma basal cortisol $^{(31)}$. Recently, the same authors have demonstrated that incubating HK from seabass (Dicentrarchus labrax), using the same superfusion system and the same conditions, with free LNA increased significantly cortisol release after ACTH stimulation (D Montero, D NegrínBáez, R Ganga, A Navarro, M Izquierdo and JM Afonso, unpublished results). In addition, it was demonstrated that reducing LNA in diets for postmenopausal women reduced the cortisol release during stress ${ }^{(62)}$. The present results are in accordance with these reports indicating the opposite effect of LA in fish fed SO in reducing cortisol release, while LNA increased this response in fish fed LO.

The mechanisms by which these fatty acids could modulate cortisol release in fish are still a subject of many research studies, and many hypotheses have been suggested through different pathways. Some reports suggested that the effect of LNA and LA on cortisol is mediated by protein kinase $\mathrm{C}$ (protein kinase A) and protein kinase A through cyclic AMP activation ${ }^{(59,61)}$. Interestingly, one of the most important roles of these EFA is that they provide eicosanoids precursors, a well-known series of hormones produced by the actions of the COX and LOX on these fatty acids, and modulate many physiological and immunological processes. The implication of COX metabolites in cortisol release has been proved recently by in vivo studies in fish ${ }^{(49-51)}$, and in our previous in vitro study, we demonstrated that incubating HK tissue with a COX inhibitor significantly decreased cortisol release $^{(20)}$. In mammals, there is clear evidence that PG modulate the release of hypothalamic corticotrophinreleasing hormone and/or pituitary $\mathrm{ACTH}^{(63-65)}$. For example, it is known that $\mathrm{PG}$, and particularly $\mathrm{PGE}_{2}$, modulate the sensitivity of the mammalian hypothalamus-pituitary-adrenal axis and consequently change the stress response ${ }^{(22,66)}$. COX-derived PG have been shown to increase in vitro cortisol release in inter-renal tissue of female frogs during ovulation ${ }^{(67)}$ as well as in human adrenal cells ${ }^{(68)}$. Elsewhere, feeding sea bream with diets containing $100 \%$ of $\mathrm{VO}$ as a blend of linseed (58\%), rapeseed (17\%) and palm (25\%) oils decreased significantly plasma $\mathrm{PGE}_{3}^{(69)}$.

In the present study, incubating $\mathrm{HK}$ tissue with INDO decreased cortisol release in FO-, 70LO-, 100LO- and 100SO-fed fish, proving the implication of prostanoids in cortisol release in these fish, and this is in accordance with our previous results ${ }^{(20)}$. The implication of LOX metabolites in cortisol release was recently investigated, but as far as we are aware, the present study is the first report of the implication of LOX derivatives in the modulation of cortisol secretion by $\mathrm{HK}$ in fish. Incubating the HK tissue with NDGA decreased the cortisol release in the majority of the treatments. This is in accordance with the results from other studies proposing the implication of the LOX pathway in stimulating ACTH release when 
ARA was added to fish cells in vitro ${ }^{(50)}$ and that LOX metabolites of ARA were reported to stimulate growth hormone release in rat anterior pituitary cells ${ }^{(63,70)}$. More studies have pointed out the role of LOX products in ACTH secretion and adrenal steroidogenesis in mammals ${ }^{(71,72)}$. In addition, other eicosanoids such as epoxygenase metabolites could also be potential modulators of the hypothalamus-pituitary-inter-renal axis in fish, since they have been demonstrated to stimulate ACTH and endorphin secretion from rat pituitary cells ${ }^{(70,73,74)}$.

In conclusion, studies carried out with fatty acids require great care in interpretation. There is a clear suggestion that changing the ratios between $n-3$ and $n-6$ fatty acids in sea bream diets by including $\mathrm{LO}$ and/or SO as substitutes to FO may alter seriously the composition of fish HK membranes ${ }^{(25-28)}$ and consequently modulate their response to stress ${ }^{(31)}$. It is clear that feeding these VO affected cortisol release in HK from gilthead sea bream, and this is mediated partly by the action of COX and LOX metabolites. Moreover, the presence of higher levels of C18 fatty acids in the HK from fish fed VO could activate other unknown pathways, and more studies are needed to clarify these mechanisms responsible for cortisol release in fish.

\section{Acknowledgements}

The present work was funded by the Spanish Ministry of Sciences and Innovation as part of LINOSALUD project (AGL2004-08151-CO302). The experiments were designed according to the Animal Welfare Ethics Committee guidelines of Las Palmas University. R. G. carried out the animal experiment, performed the biochemical studies and cortisol analysis, participated in the interpretation of the results and drafted the manuscript. J. G. B. and D. M. participated in the design of the study and in the interpretation of the results. A. F. participated in the animal experiment design and provided the formulated animal diets. Y. V. and E. A. participated in the animal feeding and sampling. L. T. supervised the cortisol analysis and the result interpretation. $M$. S. I. conceived the study and its design, coordinated the work, and participated in the interpretation of the results and the preparation of the manuscript. All the authors read and approved the final manuscript. There are no potential conflicts of interest.

\section{References}

1. Watanabe T, Tamiya T, Oka A, et al. (1983) Improvement of dietary value of live foods for fish larvae by feeding them on $\omega 3$ highly unsaturated fatty acids and fat-soluble vitamins. Nippon Suisan Gakkai Shi 49, 471-479.

2. Watanabe T, Kitajima C \& Fujita S (1983) Nutritonal values of live organisms used in Japan for mass propagation of fish: a review. Aquaculture 34, 115-143.

3. Izquierdo MS, Watanabe T, Takeuchi T, et al. (1989) Requirement of larval red seabream Pagrus major for essential fatty acids. Nippon Suisan Gakkai Shi 55, 859-867.
4. Izquierdo MS (1996) Essential fatty acid requirements of cultured marine fish larvae. Aquac Nutr 2, 183-191.

5. Castell JD, Bell JG, Tocher DR, et al. (1994) Effects of purified diets containing different combinations of arachidonic and docosahexaenoic acid on survival, growth and fatty acid composition of juvenile turbot, Scophthalmus maximus. Aquaculture 128, 315-333.

6. Bessonart M, Izquierdo MS, Salhi M, et al. (1999) Effect of dietary arachidonic acid levels on growth and survival of gilthead sea bream Sparus aurata L. larvae. Aquaculture 179, 265-275.

7. Gurr MI \& Harwood JL (1991) Lipid Biochemistry. London: Chapman \& Hall.

8. Sargent JR, Bell JG, Bell MV, et al. (1993) The metabolism of phospholipids and polyunsaturated fatty acids in fish. In Aquaculture: Fundamental and Applied Research, Coastal and Estuarine Studies, 43rd ed., pp. 103-124 [B Lahlou and P Vitello, editors]. Washington, DC: American Geophysical Union.

9. Montero D, Tort L, Izquierdo MS, et al. (1998) Depletion of serum alternative complement pathway activity in gilthead seabream caused by $\alpha$-tocopherol and $n$-3 HUFA dietary deficiencies. Fish Physiol Biochem 18, 399-407.

10. Pickering AD \& Pottinger TG (1989) Stress responses and diseases response in salmonid fish: effects of chronic elevation of plasma cortisol. Fish Physiol Biochem 7, 253-258.

11. Donaldson EM (1981) The pituitary-interrenal axis as an indicator of stress in fish. In Stress and Fish, pp. 11-47 [AD Pickering, editor]. London: Academic Press.

12. Schreck CB, Bradford CS, Fitzpatrick MS, et al. (1989) Regulation of the interrenal tissue of fishes: non-classical control mechanisms. Fish Physiol Biochem 7, 259-265.

13. Arnold-Reed DE \& Balment RJ (1991) Atrial natriuetic factor stimulates in vivo and in vitro secretion of cortisol in teleosts. J Endocrinol 128, R17-R20.

14. Lamers AE, Flik G, Atsma W, et al. (1992) A role for di-acetyl $\alpha$-melanocyte-stimulating hormone in the control of cortisol release in the teleost Oreochromis mossambicus. $J$ Endocrinol 135, 285-292.

15. Patiño R, Bradford CS \& Shreck CB (1986) Adenylate cyclase activators and inhibitors, cyclic nucleotide analogs, and phosphatidylinositol: effects on interrenal function of coho salmon (Oncorbynchus kisutch) in vitro. Gen Comp Endocrinol 63, 230-235.

16. Schimmer BP (1995) The 1994 Upjohn Award Lecture. Molecular and genetic approaches to the study of signal transduction in the adrenal cortex. Can J Physiol Pharmacol 73, 1097-1107.

17. Kloas W, Reinecke M \& Hanke W (1994) Role of the atrial natriuretic peptide for adrenal regulation in the teleost fish Cyprinus carpio. Am J Physiol 267, R1034-R1042.

18. Bird IM, Walker SW \& Williams BC (1990) Agonist-stimulated turnover of the phosphoinositides and the regulation of adrenocortical steroidogenesis. J Mol Endocrinol 5, 191-209.

19. Lacroix M \& Hontela A (2001) Regulation of acute cortisol synthesis by cAMP-dependant protein kinase and protein kinase $\mathrm{C}$ in teleost species, the rainbow trout (Oncorbynchus mykiss). J Endocrinol 169, 71-78.

20. Ganga R, Tort L, Acerete L, et al. (2006) Modulation of ACTH-induced cortisol release by polyunsaturated fatty acids in interregnal cells from gilthead seabream, Sparus aurata. J Endocrinol 190, 39-45.

21. Lands WEM (1991) Biosynthesis of prostaglandins. Annu Rev Nutr 11, 41-60.

22. Nye EJ, Hockings GI, Grice JE, et al. (1997) Aspirin inhibits vasopressin-induced hypothalamic-pituitary-adrenal activity in normal humans. J Clin Endocrinol Metab 82, 812-817. 
23. Gupta OP, Lahlou B, Botella J, et al. (1985) In vivo and in vitro studies on the release of cortisol from interrenal tissue in trout. I. Effects of ACTH and prostaglandins. Exp Biol 43, 201-212.

24. Torstensen BE, Lie O \& Froyland L (2000) Lipid metabolism and tissue composition in Atlantic salmon (Salmo salar L.) effects of capelin oil, palm oil, and oleic acid-enriched sunflower oil as dietary lipid sources. Lipids 35, 653-664.

25. Bell JG, McEvoy J, Tocher DR, et al. (2001) Replacement of fish oil with rapessed oil in diets of Atlantic salmon (Salmo salar) affects tissue lipid composition and hepatocyte fatty acid metabolism. J Nutr 131, 1535-1543.

26. Bell JG, Henderson RJ, Tocher DR, et al. (2002) Substituting fish oil with crude palm oil in the diet of Atlantic salmon (Salmo salar) affects tissue fatty acid compositions and hepatic fatty acid metabolism. J Nutr 132, 222-230.

27. Caballero MJ, Obach A, Rosenlund G, et al. (2002) Impact of different dietary lipid sources on growth, lipid digestibility, tissue fatty acid composition and histology of rainbow trout, Oncorbynchus mykiss. Aquaculture 214, $253-271$

28. Izquierdo MS, Obach A, Arantzamendi L, et al. (2003) Dietary lipid sources for seabream and seabass: growth performance, tissue composition and flesh quality. Aquac Nutr 9, 397-407.

29. Izquierdo MS, Montero D, Robaina L, et al. (2005) Alterations in fillet fatty acid profile and flesh quality in gilthead seabrem (Sparus aurata) fed vegetable oils for a long term period. Recovery of fatty acid profiles by fish oil feeding. Aquaculture 250, 431-444.

30. Montero D, Robaina L, Caballero MJ, et al. (2005) Growth, feed utilization and flesh quality of European sea bass (Dicentrarchus labrax) fed diets containing vegetable oils. A time-course study on the effect of a re-feeding period with a $100 \%$ fish oil. Aquaculture 248, 121-134.

31. Montero D, Kalinowski T, Obach A, et al. (2003) Vegetable lipid sources for gilthead seabream (Sparus aurata): effects on fish health. Aquaculture 225, 353-370.

32. Balfry SK \& Higgs DA (2001) Influence of dietary lipid composition on the immune system and disease resistance of finfish. In Nutrition and Fish Health, pp. 213-234 [C Lim and WY Cheung, editors]. New York: Haworth Press.

33. Tocher DR, Bell JG, Henderson RJ, et al. (2000) The effect of dietary linseed and rapeseed oils on polyunsaturated fatty acid metabolism in Atlantic salmon (Salmo salar) undergoing parr-smolt transformation. Fish Physiol Biochem 23, 59-73.

34. Kalogeropoulos N, Alexis MN \& Henderson RJ (1992) Effect of dietary soybean and cod-liver oil levels on growth and body composition of gilthead seabream (Sparus aurata). Aquaculture 104, 293-308.

35. Folch J, Lees M \& Sloane-Stanley GH (1957) A simple method for the isolation and purification of total lipids from animal tissues. $J$ Biol Chem 226, 497-509.

36. Christie WW (1982) Lipid Analysis. Oxford: Pergamon.

37. Rotllant J, Balm PHM, Pérez-Sánchez J, et al. (2001) Pituitary and interrenal function in gilthead sea bream (Sparus aurata $\mathrm{L}$., Teleosteí) after handling and confinement stress. Gen Comp Endocrinol 121, 333-342.

38. Rotllant J, Balm PHM, Ruane NM, et al. (2000) Pituitary proopiomelanocortin-derived peptides and hypothalamicpituitary-interrenal axis activity in gilthead sea bream (Sparus aurata) during prolonged crowding stress: differential regulation of adrenocorticotropin hormone and melanocytestimulating hormone release by corticotrophin-releasing hormone and thyrotropin-releasing hormone. Gen Comp Endocrinol 119, 152-163.
39. Rotllant J, Balm PHM, Wendelaar Bonga SE, et al. (2000) A drop in ambient temperature results in a transient reduction of interrenal ACTH responsiveness in the gilthead sea bream (Sparus aurata L.). Fish Physiol Biochem 23, $265-273$.

40. Shahidi F \& Hong C (1991) Role of metal ions and heme pigments in autoxidation of heat-processed meat products. Food Chem 42, 339-346.

41. Sokal RR \& Rolf SJ (1995) Biometry: the principles and practice of statistics. In Biological Research, 3th ed., p. 419 New York: WH Freeman and Company.

42. Sargent JR, Henderson RJ \& Tocher DR (1989) The lipids. In Fish Nutrition, 2nd ed., pp. 153-218 [JE Halver, editor]. San Diego, CA: Academic Press.

43. Henderson RJ \& Tocher DR (1987) The lipid composition and biochemistry of freshwater fish. Prog Lipid Res 26, 281-347.

44. Bell JG, Tocher DR, Farndale BM, et al. (1998) Growth, mortality, tissue histopathology and fatty acid compositions, eicosanoid production and response to stress, in juvenile turbot fed diets rich in gamma-linolenic acid in combination with eicosapentaenoic acid or docosahexaenoic acid. Prostaglandins Leukot Essent Fatty Acids 58, 353-364.

45. Waagbo R, Sandnes K, Jorgensen J, et al. (1993) Health aspects of dietary lipid sources and vitamin E in Atlantic salmon (Salmo salar). II. Spleen and erythrocyte phospholipid fatty acid composition, nonspecific immunity and disease resistance. Fisk Dir Skr Ser Ernering 6, 63-80.

46. Sargent JR, Tocher DR \& Bell JG (2002) The lipids. In Fish Nutrition, 3th ed., pp. 181-257 [JE Halver and RW Hardy, editors]. San Diego, CA: Academic Press.

47. Barton BA \& Iwama GK (1991) Physiological changes in fish from stress in aquaculture with emphasis on the response and effects of corticosteroids. Annu Rev Fish Dis 1, 3-26.

48. Mommsen TP, Vijayan MM \& Moon TW (1999) Cortisol in teleosts: dynamics, mechanism of action and metabolic regulation. Rev Fish Biol Fish 9, 211-268.

49. Koven WM, Barr Y, Lutzky S, et al. (2001) The effect of dietary arachidonic acid (20:4n-6) on growth, survival and resistance to handling stress in gilthead seabream (Sparus aurata) larvae. Aquaculture 193, 107-122.

50. Koven WM, Van Anholt RD, Lutzky S, et al. (2003) The effect of dietary arachidonic acid on growth, survival, and cortisol levels in different-age gilthead seabream larvae (Sparus aurata) exposed to handling of daily salinity change. Aquaculture 228, 307-320

51. Van Anholt RD, Spanings FAT, Koven WM, et al. (2004) Dietary supplementation with arachidonic acid in tilapia (Oreochromis mossambicus) reveals physiological effects not mediated by prostaglandins. Gen Comp Endocrinol 139, 215-226.

52. Kanazawa A (1997) Effects of docosahexaenoic acid and phospholipids on stress tolerance of fish. Aquaculture $\mathbf{1 5 5}$, 129-134.

53. Harel M, Gavasso S, Leshin J, et al. (2001) The effect of tissue docosahexaenoic and arachidonic acids levels on hypersaline tolerance and leucocyte composition in striped bass (Morone saxatilis) larvae. Fish Physiol Biochem 24, $113-123$.

54. Liu J, Caballero MJ, Izquierdo M, et al. (2002) Necessity of dietary lecithin and eicosapentaenoic acid for growth, survival, stress resistance and lipoptotein formation in gilthead seabream Sparus aurata. Fish Sci 68, 1165-1172.

55. Sumida C (1995) Fatty acids: ancestral ligands and modern co-regulators of the steroid hormone receptor cell signalling pathway. Prostaglandins Leukot Essent Fatty Acids 52, $137-144$. 
56. Vallette G, Sumida C, Thobie N, et al. (1995) Unsaturated fatty acids synergistically enhance glucocorticoid-induced gene expression. Cell Signal 7, 319-323.

57. Ballou LR \& Cheung WY (1985) Inhibition of human platelet phospholipases A2 activity by unsaturated fatty acids. Proc Natl Acad Sci U S A 82, 371-375.

58. Sarel I \& Widmaier EP (1995) Stimulation of steroidogenesis in cultured rat adrenocortical cells by unsaturated fatty acids. Am J Physiol 268, R1484-R1490.

59. Matthys LA \& Widmaier EP (1998) Fatty acids inhibit adrenocorticotropin induced adrenal steroidogenesis. Horm Metab Res 30, 80-83.

60. Yehuda S, Rabinovitz S, Carasso RL, et al. (2000) Fatty acid mixture counters changes in cortisol, cholesterol and impair learning. Int J Neurosci 101, 73-87.

61. Ando H, Oka M, Ichihashi M, et al. (1990) Protein kinase C and linoleic acid-induced inhibition of melanogenesis. Pigment Cell Res 3, 200-206.

62. Spence JD, Thornton T, Muir AD, et al. (2003) The effect of flax seed cultivars with differing content of $\alpha$-linolenic acid and lignans on response to mental stress. J Am Coll Nutr 22, 494-501.

63. Abou-Samra AB, Catt KJ \& Aguilera G (1986) Role of arachidonic acid in the regulation of adreno-corticotropin release from rat anterior pituitary cell cultures. Endocrinology 119, 1427-1431.

64. Zacharieva S, Borissova AM, Andonova K, et al. (1992) Role of prostaglandin $\mathrm{E} 2\left(\mathrm{PGE}_{2}\right)$ on the corticotropin-releasing hormone (CRH)-induced ACTH release in healthy men. Horm Metab Res 24, 336-338.

65. Nasushita R, Watanobe H \& Takebe K (1997) A comparative study of adrenocorticoropin-releasing activity of prostaglandins $\mathrm{E}_{1}, \mathrm{E}_{2}, \mathrm{~F}_{2 \alpha}$ and $\mathrm{D}_{2}$ in the rat. Prostaglandins Leukot Essent Fatty Acids 56, 165-168.
66. Bugajski J, Gadek-Michalska A \& Bugajski AJ (2001) A single corticosterone pre-treatment inhibits the hypothalamicpituitary-adrenal responses to adrenergic and cholinergic stimulation. J Physiol Pharmacol 52, 313-324.

67. Gobbetti A \& Zerani M (1993) Prostaglandin $E_{2}$ and prostaglandin $\mathrm{F}_{2}$ alpha involvement in the corticosterone and cortisol release by the female frog, Rana esculenta, during ovulation. J Exp Zool 267, 164-170.

68. Vakharia K \& Hinson JP (2005) Lipopolysacharide directly stimulates cortisol secretion by human adrenal cells by a cyclooxygenase-dependent mechanism. Endocrinology 146, 1398-1402.

69. Ganga R, Bell JG, Montero D, et al. (2005) Effect of dietary lipids on plasma fatty acid profiles and prostaglandin and leptin production in gilthead seabream (Sparus aurata). Comp Biochem Physiol B Biochem Mol Biol 142, 410-418.

70. Won JGS \& Orth DN (1994) Role of lipoxygenase metabolites of arachidonic acid in the regulation of adrenocorticotropin secretion by perfused rat anterior pituitary cells. Endocrinology 135, 1496-1503.

71. Wang H, Walker SW, Mason JI, et al. (2000) Role of arachidonic acid metabolism in ACTH-stimulated cortisol secretion by bovine adrenocortical cells. Endocr Res 26, 705-709.

72. Yamazaki T, Higuchi K, Kominami S, et al. (2001) 15-Lipoxygenase metabolite(s) of arachidonic acid mediates adrenocorticotropin action in bovine adrenal steroidogenesis. Endocrinology 137, 2670-2675.

73. Michl P, Beikler T, Engelhardt D, et al. (2000) Interleukin-3 and Interleukin- 6 stimulate bovine adrenal cortisol secretion through different pathways. J Neuroendocrinol 12, 23-28.

74. Cowell AM, Flower RJ \& Buckingham JC (1991) Studies on the roles of phospholipase $\mathrm{A}_{2}$ and eicosanoids in the regulation of corticotrophin secretion by rat pituitary cells in vitro. J Endocrinol 130, 21-32. 